\title{
Proximity Ligation Assay
}

National Cancer Institute

\section{Source}

National Cancer Institute. Proximity Ligation Assay. NCI Thesaurus. Code C156754.

A technique that leverages immunoassay and DNA amplification technology to detect protein-protein interactions with high specificity and sensitivity. A sample is prepared and then exposed to primary antibodies raised in different species recognizing the target epitopes on the proteins of interest. Then, secondary antibodies that are tagged with short oligonucleotides and that target each primary antibody are added. Next, a mixture comprised of a ligase, PCR components and a connector oligonucleotide, which hybridizes to both oligonucleotide tags and can prime rolling DNA circle synthesis, are added and the sample is subjected to real-time PCR to amplify the DNA circles. Rolling circle synthesis and amplification is only possible if the protein epitopes are in close proximity (i.e. in a protein complex). Finally, a labeled complementary nucleotide probe is added to detect and/or visualize the amplified DNA. 